\title{
Targeting the Microvasculature in Cerebral Ischemia-Go (or Stop Going) with the Flow
}

\author{
J. Marc Simard • Volodymyr Gerzanich
}

Received: 22 March 2011 / Accepted: 22 March 2011 / Published online: 14 April 2011

(C) Springer Science+Business Media, LLC 2011

In this issue of Translational Stroke Research, Turner et al. [1] address the important question of the relevance of the model(s) of stroke that are used in preclinical animal studies. They argue, quite rightly, that our heavy reliance on rodent models, especially rats, may be inadequate for predicting what will work in humans. This sentiment echoes the recommendations of the Stroke Treatment Academic Industry Roundtable (STAIR), which suggests that treatment efficacy should be established in at least two species, with initial testing being carried out in rodents or rabbits [2]. Turner et al. argue specifically for broader use of the rabbit embolic clot model. The essence of their argument favoring the use of the rabbit embolic clot model is twofold: first, the only pharmacological treatment currently approved for use in humans is recombinant tissue plasminogen activator (rtPA), which acts on an embolic clot; second, the use of rtPA in the rabbit embolic clot model accurately predicts the success of rtPA in humans. The demonstrated predictive utility of the rabbit embolic clot model, they argue, is likely to generalize to other pharmacological treatments for stroke.

There is merit to the argument of Turner et al. Beyond this, however, their argument raises a broader issue that is frequently overlooked - the only successful therapeutic target thus far identified in stroke is the cerebral vasculature. Without a sufficient, sustainable source of nutrients, which are carried exclusively by a functional microvasculature, the neuron is doomed. Without a properly functioning microvasculature, the neuron will never receive the newest miracle neuro-

\footnotetext{
J. M. Simard $(\bowtie) \cdot$ V. Gerzanich

Neurosurgery Department,

University of Maryland School of Medicine,

22 S. Greene St., Suite S12D,

Baltimore, MD 21201-1595, USA

e-mail: msimard@smail.umaryland.edu
}

protectant, be it an anti-apoptotic drug, siRNA against hypoxia inducible factor 1 [3], inhibitor peptide against nuclear factor $\mathrm{\kappa B}[4]$, etc. No one doubts the importance of the neuron, but without an intact, normally functioning vasculature, no agent can effectively protect the neuron. Virtually all preclinical and clinical tests of neuroprotectants assume a functional microvasculature. How good is this assumption? If the assumption is not valid, might this account for some of our failures?

Enormous efforts have gone into identifying and testing well over 1,000 pharmaceutical agents for neuroprotective capability [5]. For the most part, the agents tested in preclinical models are agents that have been identified in various in vitro systems, including brain slices, cell cultures, and molecular studies that are usually aimed at understanding neuronal pathophysiology initiated by ischemia/hypoxia. Even in the current "post-neuron-centric" age, in what is now the enlightened age of the neurovascular unit, efforts continue apace to identify molecular mechanisms that can be targeted to salvage neurons.

By contrast, the cerebral microvascular endothelium is neglected. Few "protective" agents specifically target the microvasculature in ischemia/hypoxia. After ischemia/hypoxia, the microvasculature is never normal, but suffers one of two broad fates: (1) it can become occluded due to endothelial cell swelling, with no restoration of flow [6] and (2) flow may be partial but impeded due to endothelial cell swelling, with the endothelial cell becoming pathologically dysfunctional [7]. In the first instance, with flow occlusion, no neuroprotectant can be conceived that will reach the neuron to save it. In the second instance, impeded flow via abnormal dysfunctional capillaries gives rise to harmful pathological processes. Capillary endothelial cells subjected to ischemia/hypoxia undergo not only swelling (cytotoxic edema), which compromises microcirculatory flow [6], but 
in addition, they lose the integrity of inter-endothelial tight junctions, which allows extravasation of plasma and promotes the formation of vasogenic edema [7]. Ultimately, complete loss of the structural integrity of capillaries can lead to the formation of petechial hemorrhages and hemorrhagic transformation [7]. If the brain and the organism survive the edema and the hemorrhagic transformation, the activated capillary endothelial cell becomes the gateway for inflammatory cells, which cause additional injury.

The argument set forth by Turner et al., as well as by STAIR and many others, is that our heavy reliance on rodent models, especially rats, may be inadequate for predicting what will work in humans. Perhaps we should consider rephrasing the argument: our heavy reliance on neuronal cells in vitro to first identify molecular targets may be inadequate for predicting what will work in humans in vivo.

Should more efforts be devoted to understanding endothelial pathophysiology induced by ischemia/hypoxia? We believe the answer is an unequivocal "yes," but there are caveats. Unfortunately, cultured endothelial cells provide only a limited solution. The phenotype found in vitro only partially recapitulates the phenotype in vivo. Unfortunately, endothelial cells in vivo are extremely difficult to study. We have no convenient way to study the physiology of intact functional microvessels, although advances in twophoton microscopy [8-10] may remove some barriers. Thus far, relevant advances have been limited. Although work on matrix metalloproteinases [11, 12] and tight junction proteins [13], among others [14, 15], are extremely promising, they have yet to advance to clinical translation.

In the end, Turner et al. are correct. The fact that rtPA is the only pharmacological treatment currently approved for use in humans, and that the use of rtPA in the rabbit embolic clot model accurately predicts the success of rtPA in humans, conveys one or more very potent messages. One message is that the vasculature is the only successful target thus far identified. In some ways, the problem for the major conduit vessels has been "solved," by restoring flow with thrombolytics or by mechanical means. However, the microvasculature has been neglected, and we have only begun to identify relevant molecular targets induced in the microvasculature by ischemia/hypoxia.

The phrase, "Go with the Flow," is first known to have been used by the Roman Emperor Marcus Aurelius in his writings, "the Meditations." Marcus wrote about the flow of thoughts and happiness. He concluded that "most things flow naturally" and in his opinion, it was better to "Go with the Flow" than to try and change society. Today, the phrase still carries the same meaning: "to not push against prevailing behavior, norms or attitudes." In this commentary, we use the phrase differently to underscore the importance of maintaining normal microvascular flow after cerebral ischemia-in this sense, do "go with the flow." As a result, however, we advocate in favor of greater focused study of the pathophysiology induced by hypoxia/ischemia, but not in the neuron, rather in the cerebral microvasculature - in this sense, "stop going with the flow."

\section{References}

1. Turner RJ, Jickling GC, Sharp FR. Are underlying assumptions od current animal models of human stroke correct: from STAIRS to high hurdles? Trans Stroke Res. 2011;2(2). doi:10.1007/s12975011-0067-3.

2. Fisher M, Feuerstein G, Howells DW, Hurn PD, Kent TA, Savitz SI, et al. Update of the stroke therapy academic industry roundtable preclinical recommendations. Stroke. 2009;40:2244-50.

3. Yeh SH, Ou LC, Gean PW, Hung JJ, Chang WC. Selective inhibition of early - but not late-expressed HIF-1alpha is neuroprotective in rats after focal ischemic brain damage. Brain Pathol. 2011 (in press).

4. Desai A, Singh N, Raghubir R. Neuroprotective potential of the NF-kappaB inhibitor peptide IKK-NBD in cerebral ischemiareperfusion injury. Neurochem Int. 2010;57:876-83.

5. O'Collins VE, Macleod MR, Donnan GA, Horky LL, van der Worp BH, Howells DW. 1,026 experimental treatments in acute stroke. Ann Neurol. 2006;59:467-77.

6. Hills CP. Ultrastructural changes in the capillary bed of the rat cerebral cortex in anoxic-ischemic brain lesions. Am J Pathol. 1964;44:531-51.

7. Simard JM, Kent TA, Chen M, Tarasov KV, Gerzanich V. Brain oedema in focal ischaemia: molecular pathophysiology and theoretical implications. Lancet Neurol. 2007;6:258-68.

8. Park HP, Nimmagadda A, DeFazio RA, Busto R, Prado R, Ginsberg MD. Albumin therapy augments the effect of thrombolysis on local vascular dynamics in a rat model of arteriolar thrombosis: a two-photon laser-scanning microscopy study. Stroke. 2008;39:1556-62.

9. Lam CK, Yoo T, Hiner B, Liu Z, Grutzendler J. Embolus extravasation is an alternative mechanism for cerebral microvascular recanalization. Nature. 2010;465:478-82.

10. Jung JC, Mehta AD, Aksay E, Stepnoski R, Schnitzer MJ. In vivo mammalian brain imaging using one- and two-photon fluorescence microendoscopy. J Neurophysiol. 2004;92:3121-33.

11. del Zoppo GJ. The neurovascular unit, matrix proteases, and innate inflammation. Ann NY Acad Sci. 2010;1207:46-9.

12. Morancho A, Rosell A, Garcia-Bonilla L, Montaner J. Metalloproteinase and stroke infarct size: role for anti-inflammatory treatment? Ann NY Acad Sci. 2010;1207:123-33.

13. Jiao H, Wang Z, Liu Y, Wang P, Xue Y. Specific role of tight junction proteins claudin-5, occludin, and ZO-1 of the blood-brain barrier in a focal cerebral ischemic insult. J Mol Neurosci. 2011 (in press).

14. Chiba Y, Sasayama T, Miyake S, Koyama J, Kondoh T, Hosoda $\mathrm{K}$, et al. Anti-VEGF receptor antagonist (VGA1155) reduces infarction in rat permanent focal brain ischemia. Kobe J Med Sci. 2008;54:E136-46.

15. Lee SW, Kim WJ, Jun HO, Choi YK, Kim KW. Angiopoietin-1 reduces vascular endothelial growth factor-induced brain endothelial permeability via upregulation of ZO-2. Int $\mathrm{J}$ Mol Med. 2009;23:279-84. 